NEWS

\title{
A shot in the arm for cancer vaccines?
}

\section{Researchers anxiously await a decision by US regulators on a controversial cancer therapy.}

In the early 1990s, immunologist Edgar Engleman of California's Stanford University School of Medicine thought he had discovered a way to treat cancer using a vaccine that harnessed the body's immune cells. He co-founded a company — later named Dendreon - in 1992 to develop the vaccine, predicting that it would reach patients within a few years. "We were so naïve," he says. "We didn't know what to expect."

Now, after some 20 years of successes and setbacks, Dendreon's prostate-cancer vaccine Provenge (sipleucel-T) may finally be nearing the market; the US Food and Drug Administration (FDA) is expected to reach a decision on its approval by 1 May. If the vaccine is approved, it will mark a turning point for the field of therapeutic cancer vaccines, an approach that seemed promising but developed a disappointing reputation after several high-profile failures in clinical trials. Although a few vaccines have been licensed for use in other countries, none has broken through to the US market. An FDAapproved vaccine, says Theresa Whiteside, an immunologist at the University of Pittsburgh School of Medicine in Pennsylvania, "would sort of legitimize the field". It would also offer a potential new treatment for patients with advanced prostate cancer, which killed more than 28,000 US men in 2008.

Provenge is much more complex than familiar vaccines against viruses, such as measles or human papilloma virus, the cause of most cervical cancers. The vaccine is tailor-made for each patient by harvesting his dendritic cells - a type of immune cell - and exposing them to a cancer-associated protein called prostatic acid phosphatase. Once infused back into the patient, the exposed cells should

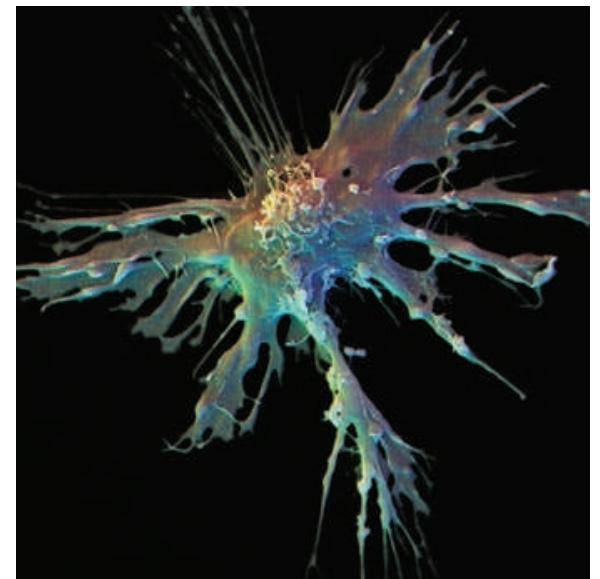

A vaccine using dendritic cells might be approved.

trigger an immune assault on tumour cells.

The vaccine seemed to be on the cusp of approval three years ago, after an FDA advisory committee determined that it was both safe and effective for use in advanced prostate cancer. But the FDA had lingering concerns, noting that Dendreon's phase III clinical trials were relatively small. What's more, although Provenge extended the lifespan of men with advanced prostate cancer, it did not slow tumour growth, the endpoint that those trials were designed to address. In a highly controversial decision, the agency ordered Dendreon, headquartered in Seattle, Washington, to complete a further large clinical trial in 500 patients, this time designating overall survival as the trial's endpoint. The results of that trial, released last April, showed that Provenge lengthened the survival of patients with late-stage prostate cancer by four months.

Provenge has won the fervent support of patients with prostate cancer as well as their advocates, and the company's rising stock price (see 'Wild ride for a cancer treatment') is evidence that investors are optimistic about the vaccine's approval. However, it promises to be expensive. Dendreon has not yet set a price, but some analysts estimate that it will cost up to US $\$ 100,000$ per patient. Meanwhile, many researchers are reserving judgement on its efficacy until Dendreon publishes the results of its latest clinical trial in a peer-reviewed journal.

There are also questions over exactly how the vaccine works. Provenge is a relatively crude mixture of different cell types, including the dendritic cells that should stimulate the immune response. "It would be nice to know exactly what's in there and what these other cells are contributing," says Nina Bhardwaj, an immunologist at New York University's Langone Medical Center in New York.

Nevertheless, given that Provenge relies on nearly 20 -year-old technology, the vaccine's performance is impressive, says Bhardwaj. Many first-generation cancer vaccines such as PANVAC, a pancreatic cancer vaccine, were deemed safe but failed to demonstrate that they significantly slowed the progression of cancer. Because cancer-associated antigens - such as those used in Provenge - are also found at low levels in healthy tissue, their ability to trigger a powerful immune response may be blunted.

A second generation of vaccines, designed to provoke a stronger immune response, is under development, with some scientists now focusing on antigens that are found only on tumour cells. One of the first vaccines to use this approach targets a mutant protein called EGFRvIII that is found in glioblastoma, an

\section{WILD RIDE FOR A CANCER TREATMENT}

For almost 20 years, Dendreon has been developing a vaccine for prostate cancer. Hopes for the vaccine, called Provenge, have fluctuated over the years along with Dendreon's stock price.

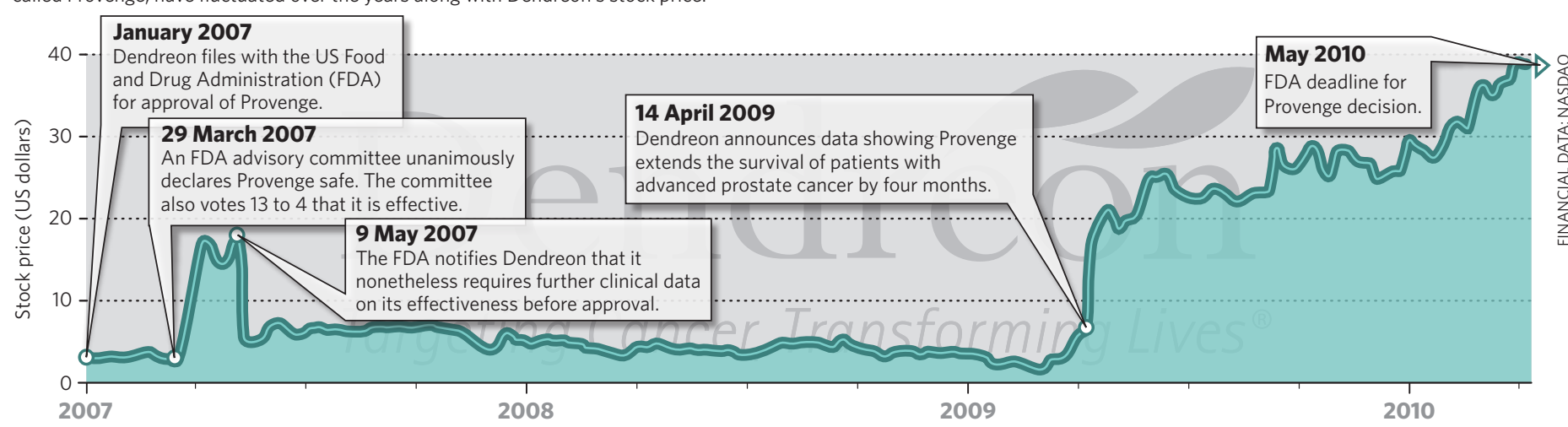


aggressive brain cancer. The vaccine is being jointly developed by drugs giant Pfizer, based in New York, and Celldex, a biotechnology firm headquartered in Needham, Massachusetts.

Over the past decade, researchers have reached a deeper understanding of how tumours actively suppress immune responses in their immediate environment, which can dampen responses to cancer vaccines. To overcome this, some therapies currently in development combine the vaccine with chemotherapies that are designed to counteract this immune suppression. For example, a Seattle-based biotechnology company called Oncothyreon has developed a cancer vaccine called Stimuvax that is administered in combination with the drug cyclophosphamide. The compound inhibits immune cells called T-regulatory cells, which block immune responses to the body's own molecules.

Compounds that modulate the immune response could have unwanted side effects, however. A patient in a clinical trial of Stimuvax involving high doses of cyclophosphamide developed an acute inflammation of the brain, which caused the FDA to put all Stimuvax trials on hold.

A clean safety profile is crucial if cancervaccine developers are to improve a vaccine's performance in clinical trials. To date, most of these trials have enrolled patients who are in the advanced stages of cancer, which may have limited the trials' effectiveness because such individuals may not be able to mount an effective immune response. Now that such vaccines have been established as safe in phase II trials, clinicians are more willing to test them in healthier patients. An ongoing large trial of a lung cancer vaccine by London-based pharmaceutical firm GlaxoSmithKline, for example, is enrolling patients at an earlier stage of the disease.

For some in the field, the struggle to create effective cancer vaccines conjures up memories of the long battle to develop antibody-based therapies, which are now a mainstay of the biotechnology industry. There, too, a series of clinical-trial failures initially soured the field's reputation, recalls Thomas Davis, chief medical officer at Celldex. In the early 1990s, when Davis worked to develop rituximab - a monoclonal antibody used to treat autoimmune disorders and some cancers - he recalls that researchers in the field learned to be resilient. "We realized you just have to test a lot of drugs to find one that works," he says, "and it's the same for a cancer vaccine." Heidi Ledford

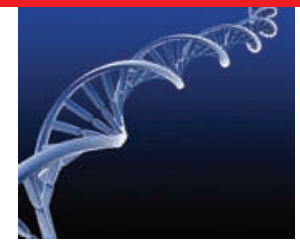

A HISTORY OF METHYLATION

Analysis tracks evolution of DNA modification process. go.nature.com/GUa4b2

\section{No gain from brain training}

The largest trial to date of 'brain-training' computer games suggests that people who use the software to boost their mental skills are likely to be disappointed.

The study, a collaboration between British researchers and the BBC Lab UK website, recruited viewers of the $\mathrm{BBC}$ science programme Bang Goes the Theory to practise a series of online tasks for a minimum of ten minutes a day, three times a week, for six weeks. In one group, the tasks focused on reasoning, planning and problem-solving abilities - skills correlated with general intelligence. A second group was trained on mental functions targeted by commercial brain-training programs - short-term memory, attention, visuospatial abilities and maths. A third group, the control subjects, simply used the Internet to find answers to obscure questions. A total of 11,430 volunteers aged from 18 to 60 completed the study, and although they improved on the tasks, the researchers believe that none of the groups boosted their performance on tests measuring general cognitive abilities such as memory, reasoning and learning.

"There were absolutely no transfer effects" from the training tasks to more general tests of cognition, says Adrian Owen, a neuroscientist at the Medical Research Council (MRC) Cognition and Brian Sciences Unit in Cambridge, UK, who led the study. "I think the expectation that practising a broad range of cognitive tasks to get yourself smarter is completely unsupported."

It's unlikely that the study, published online in Nature this week ${ }^{1}$, will quell the brain-training debate. "I really worry about this study - I think it's flawed," says Peter Snyder, a neurologist who studies ageing at Brown University's Alpert Medical School in Providence, Rhode Island. Snyder agrees that data supporting the efficacy of brain training are sparse. Although some earlier studies - such as one ${ }^{2}$ funded by Posit Science, a brain-training software company in San Francisco, California - showed modest effects, Snyder recently published a meta-analysis that found little benefit ${ }^{3}$.

But he says that most commercial programs are aimed at adults well over 60 who fear that their memory and mental sharpness are slipping. "You have to compare apples to apples," says Snyder. An older test group, he adds, would have a lower mean starting score and more variability in performance, leaving more room for training to cause meaningful improvement. "You may have more of an ability to see an effect if you're not trying to create a supernormal effect in a healthy person," he says.

Indeed, the subjects in this study were a self-selected group "who would have had a natural inclination to play this sort of game", says David Moore, director of the MRC Institute of Hearing Research in Nottingham, UK, and a founder of MindWeavers, a company in Oxford, UK, selling the brain-training program

\section{MindFit.}

Moore and Snyder add that the training time may not have been long enough. Subjects completed an average of 24 sessions - at ten minutes a session, that's just four hours of training, says Snyder. "Four hours of testing over six weeks isn't a lot to create meaningful change." Brain-training
reatments for lazy eye or exercises such as treatments for lazy eye or
some post-stroke training regimens require more time to work, says Moore.

Owen counters that several similar studies have used a six-week training period. Although the average number of sessions in his trial was 24 , the actual number ranged from two to "some real diehards doing it several hundred times", he says, and he saw no difference in performance between the extremes. "There is no psychological theory that could account for [no effects at all] for six weeks, and then suddenly at week 22 an effect," he says.

Owen concedes that his findings don't necessarily mean that training in young children or elderly patients is pointless. But "the evidence is not strong", he says. "And someone needs to go and test it."

Alla Katsnelson

1. Owen, A. M. et al. Nature advance online publication doi:10.1038/nature09042 (20 April 2010).

2. Smith, G. E. et al. J. Am. Geriatr. Soc. 57, 594-603 (2009)

3. Papp, K. V., Walsh, S. J. \& Snyder, P. J. Alzheimers Dement. 5, 50-60 (2009) 